\title{
Brain tumor segmentation from multimodal magnetic resonance imaging data based on gray- level co-occurrence matrix (GLCM) and an ensemble Support Vector Machine (SVM) classifier
}

$\mathrm{Na}$ Li ( $\nabla$ lina@scuec.edu.cn )

South-central university for nationalities https://orcid.org/0000-0001-9531-9003

Zheng Yang

South-central University for Nationalities

Research article

Keywords: Medical image segmentation, Brain tumor MRI, GLCM feature, an ensemble Support Vector Machine

Posted Date: August 2nd, 2020

DOI: https://doi.org/10.21203/rs.3.rs-49212/v1

License: (c) (i) This work is licensed under a Creative Commons Attribution 4.0 International License.

Read Full License 
Brain tumor segmentation from multimodal magnetic resonance imaging data based on gray-level co-occurrence matrix (GLCM) and an ensemble Support Vector Machine (SVM) classifier

\author{
$\mathrm{Na} \mathrm{Li}$ \\ Department of Computer Science, \\ South-Central University for Nationalities, Wuhan, China,430074 \\ lina@scuec.edu.cn \\ Zheng Yang \\ Department of Life Science, \\ South-Central University for Nationalities, Wuhan, China \\ 438070605@qq.com
}

\begin{abstract}
Background: Brain tumors, abnormal cells growing in the human brain, are common neurological diseases that are extremely harmful to human health. Malignant brain tumors can lead to high mortality. Magnetic resonance imaging (MRI), a typical noninvasive imaging technology, can produce high-quality brain images without damage and skull artifacts, as well as provide comprehensive information to facilitate the diagnosis and treatment of brain tumors. Additionally, the segmentation of MRI brain tumors utilizes computer technology to segment and label tumors and normal tissues automatically on multimodal brain images, which plays an important role in disease diagnosis, treatment planning, and surgical navigation.
\end{abstract}

Methods: We propose a solution using gray-level co-occurrence matrix (GLCM) texture and an ensemble Support Vector Machine (SVM) structure. We focus on the effects of GLCM texture on brain tumor segmentation. First, 112 GLCM features for each voxel were extracted. Next, these features were ranked using the SVM-recursive feature elimination (SVM-RFE) method. Based on the sorting results, we found that when the number of features was 60 , the value of the Dice similarity coefficient (DSC) tended to be flat. The GLCM texture features maximal correlation coefficient, information measure of correlation, Angular Second Moment, sum of squares, difference variance, contrast, and inverse difference moment were important for segmentation. Finally, we selected the top 60 grayscale features and constructed an ensemble SVM classifier to separate the abnormal mass of tissue from normal brain tissues.

Results: The experimental material was a dataset called BraTs2015. The proposed model was verified with the Dice coefficient. For low-grade tumors, we obtained a 91.2\% average Dice coefficient for segmenting the complete tumor region. For high-grade tumors, the average was slightly higher at $92.4 \%$.

Conclusion: Our results demonstrated that this method has a better capacity and higher segmentation accuracy with a low computation cost.

Key words: Medical image segmentation, Brain tumor MRI, GLCM feature, an ensemble Support Vector Machine

\title{
1 Background
}

Currently, a large number of digital images are generated every day in the medical field. 
Radiologists analyze and diagnose the possible lesions in the images based on their own experience. Some studies have shown that two radiologists reading a set of image data at the same time can significantly improve the detection rate of cancer, but this protocol would greatly increase the workload of doctors [1]. Computer-aided diagnosis (CAD) can be used as a second 'radiologist'. This method would improve the accuracy of disease diagnosis but not increase the doctors' workload. Furthermore, it might avoid the diagnostic errors that could result from doctors' long working hours. With a focus on brain tumors, a high incidence cancer, we proposed a texture feature representation method for images based on gray level co-occurrence matrix (GLCM), which greatly improved the distinguishability of tumors.

Texture analysis plays an important role in image processing. As an informal definition, the texture of an image can be considered the distribution pattern of grayscale in the image space, which has certain randomness and regularity [2]. The commonly used methods for describing grayscale and texture features are first-order statistics [3], GLCM [4-7], Gabor filter [8], and wavelet transform [9], among others. Haralick et al. [10] proposed using GLCM to describe the texture of an image. In this method, the neighbor relationship between pixels is defined and a GLCM is obtained. A GLCM is a compact form of the representation of the special dependency between various gray scales of an image according to a neighborhood standard that includes a distance and a direction parameter [11]. A group of 14 texture attributes based on GLCM was proposed by Haralick and colleagues [10]. They are used to describe texture in an image.

In medical image processing, there are myriad methods based on GLCM. Mayerhoefer et al. [12] proposed a new method for acquiring magnetic resonance imaging (MRI) parameters. The designed classifier is based on the GLCM feature. Alic et al. [13] proposed a GLCM-based feature to predict the treatment results of 18 kinds of limb cancer. Their reported accuracy is about $83 \%$. Agner et al. [14] designed another GLCM-based classifier that can distinguish benign and malignant breast diseases. The accuracy of their method has reached 90\%. Torheim et al. [15] proposed a method for predicting the outcome of cervical cancer treatment based on GLCM features. The accuracy of this method is $75 \%$.

For brain tumor image segmentation, the GLCM texture is used to segment the image. These methods also provide good segmentation results $[6,16]$. Indeed, most of the previous studies have selected part or all of the GLCM features. There is no one way to perform a comprehensive analysis of the 14 GLCM components in brain tumor image segmentation. An important component of our study is the influence of GLCM texture on the classification of brain tumor images. The impact of each component on the classification should be observed. This paper calculated 14 features of GLCM in four directions. These features were ranked using the SVM-recursive feature elimination (SVM-RFE) method. The experimental results showed that the maximal correlation coefficient, information measure of correlation, Angular Second Moment, sum of squares, difference variance, contrast, and inverse difference moment in GLCM were always on the top. This finding indicates that these components play a key role in brain tumor MR image segmentation. Based on the feature sorting results, we selected some features to build the SVM classifier each time. Using the test data, the full segmentation result of the SVM classifiers was tracked. We found that the value of the Dice similarity coefficient (DSC) tended to be stable when the number of features was 60 . Therefore, the top 60 features were selected. Some gray features, including gray voxel values, mean gray values, and gray value variances, on three different spatial scales and the selected GLMC features can map a high dimensional matrix. 
Ensemble SVM classifiers are proposed to detect the blurred regions in images [17]. We applied the methods developed in this paper, and successfully segmented tumor images.

\section{Methods}

The workflow of our segmentation method includes the following steps: image preprocessing, feature calculation and ensemble SVM classification (Fig. 1).

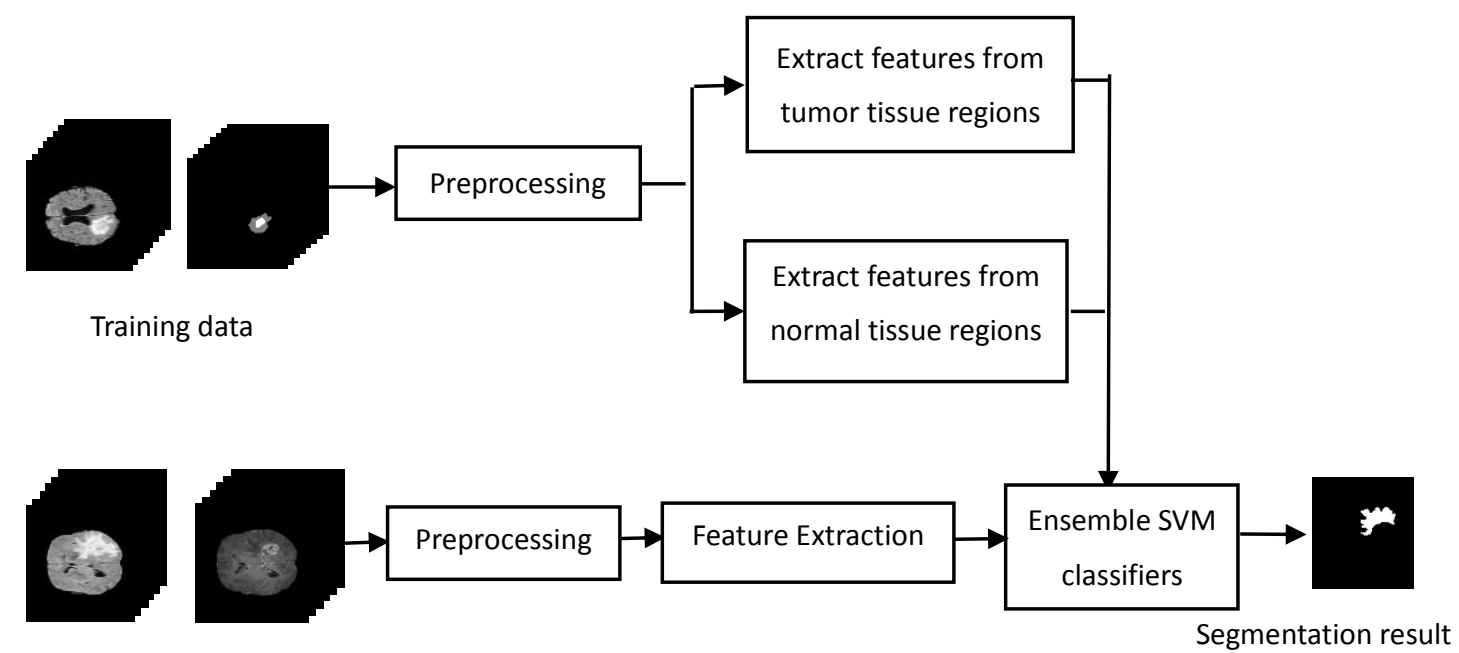

Testing data

Fig. 1. The workflow of image segmentation.

\subsection{Preprocessing}

The data comes from different devices, and thus they have different gray levels. Therefore, it is necessary to correct grayscale inconsistencies and reduce image noise. To this end, the grayscale of the MRI image was normalized from 0 to 255, and a Gaussian filter was used to reduce Gaussian noise on the image. Both steps are required during the training and testing phases. A pre-segmentation process was added during the testing phase. Pre-segmentation reduces the amount of data and greatly improves the segmentation accuracy.

Generally, the left and right hemispheres of a normal human brain are approximately symmetrical [18]. Brain tumors destroys this symmetry, a phenomenon that is reflected in the image data. The left hemisphere of the tumor image is $f_{L}$ and the right hemisphere is $f_{R}$. If $f_{L}$ is flipped along the axis of symmetry, the result is the mirror image, $f_{L M}$. If $f_{R}$ is flipped along the axis of symmetry, the mirror image is $f_{R M}$. The image differences between the left and right hemispheres are as follows:

$$
\begin{aligned}
& f_{1}=f_{L}-f_{R M} \\
& f_{2}=f_{M}-f_{L M}
\end{aligned}
$$

$f_{1}$ is the difference of the left hemisphere image, and $f_{2}$ is the difference of the right hemisphere image.

Fig. 2 presents an example of symmetry analysis of a brain image. Fig. 2a represents the original input image. It is the fluid-attenuated inversion recovery (FLAIR) modality data from MRI images. Fig. $2 \mathrm{~b}$ is the left hemisphere and Fig. $2 \mathrm{c}$ is the right hemisphere. Fig. $2 \mathrm{~d}$ presents the results calculated according to formula (1). Fig. 2e indicates the result calculated according to formula (2). The tumor area is in the right hemisphere, and thus the right hemisphere image minus the mirror image of the left 
hemisphere image is shown in Fig. 2. Tumor regions are preserved by using symmetrical information. In MRI data, the image of a brain tumor area has higher gray value in FLAIR modality. Therefore, in the pre-segmentation stage, only FLAIR modality data was used. Each patient's MRI sequences is $240 \times 240 \times 155$. There are 155 scan layers. Many experiments show that there are almost no brain tumor tissue images in the first 40 and last 30 scan images. The images of the 41 st to 125 th scan layer images were calculated according to formulae 1 and 2 . The calculation results of the 85 scanning layers were added to obtain a new image. Then, some morphological processing methods were applied to the image to complete the pre-segmentation result (Fig. $2 \mathrm{~g}$ ).

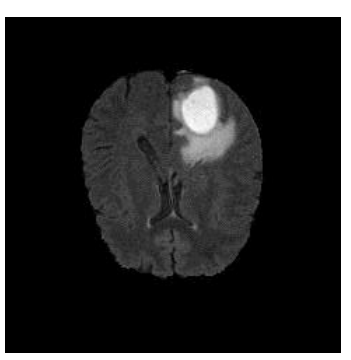

(a)

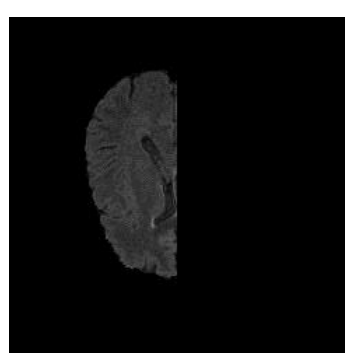

(b)

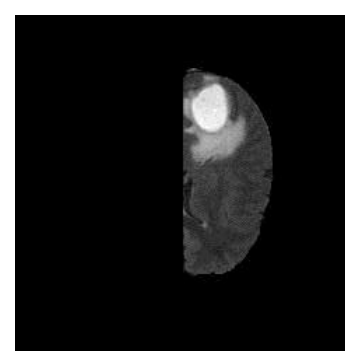

(c)

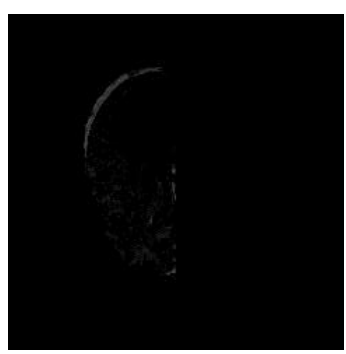

(d)

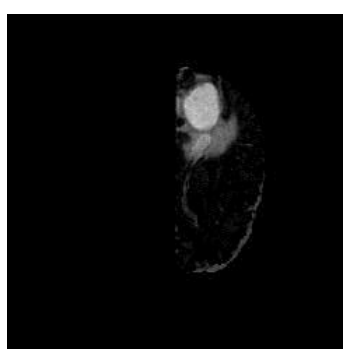

(e)

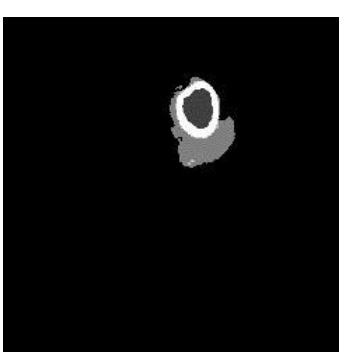

(f)

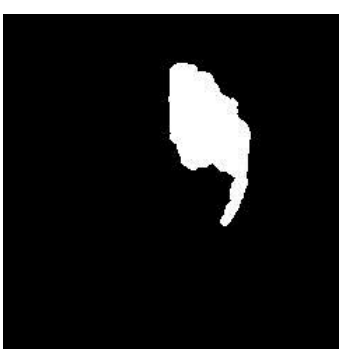

(g)

Fig. 2. Symmetric pre-segmentation of brain tumor images: (a) original image, (b) left brain image, (c) right brain image, (d) $f_{1}(x)$ image, (e) $f_{2}(x)$ image, (f) ground-truth image, and (g) pre-segmented image of all sequences in MRI.

Through many experiments, we found that pre-segmentation processing reduced the amount of data. Consequently, the training time was two-thirds shorter than the original segmentation time. The segmentation accuracy also improved to varying degrees, especially for slices with less brain tumor tissue, and its segmentation accuracy was multiplied.

\subsection{GLMC features extraction}

GLCM is one of most commonly used methods for texture feature extraction. The GLCM determines the textural relationship between pixels by performing an operation according to second-order statistics in the images [10]. Specifically, the probability of the occurrence of two pixels with a specific distance in a certain direction is calculated. This value represents the frequency formation of the pixel pairs. Haralick et al. [10] suggested 14 measures that can be extracted from each of the gray-tone spatial dependence matrices. They are as follows: Angular Second Moment, contrast, correlation, sum of squares, inverse difference moment, sum average, sum variance, sum entropy, entropy, difference variance, difference entropy, information measure of correlation, and maximal correlation coefficient. For the selected distance $d$, there are four angular grayscale spatial dependent matrices. In our experiment, we set the value of $d$ as 5. Thus, we obtained a set of four values for each of the preceding 14 measures. The mean and range of these 14 measures comprised the set of 28 features. There may be a strong correlation among these 
28 features. Moreover, it should be noted that MR imaging of brain tumor patients is a three-dimensional, multi-band imaging technique that usually includes four modalities. Thus, there are a total of 112 features. Feature selection should be applied to select a subset of the 112 features (Table 1).

Table 1. Overview of all 112 features from magnetic resonance imaging (MRI).

\begin{tabular}{lll}
\hline Feature category & Description & Corresponding index \\
\hline FLAIR MRI features & The mean and range of these 14 measures & $1-28$ \\
\hline T1 MRI features & The mean and range of these 14 measures & $29-56$ \\
\hline T1C MRI features & The mean and range of these 14 measures & $57-84$ \\
\hline T2 MRI features & The mean and range of these 14 measures & $85-112$ \\
\hline
\end{tabular}

2.3 Ensemble SVM

The SVM method, based on the statistical learning theory, presents many advantages. SVM exhibits a good generalization ability and relatively high precision even when there are relatively few samples [19]. At the same time, SVM can effectively deal with nonlinear data by introducing a kernel function. Radial basis function (RBF) kernel may be well applied in some multimodal MRI images $[20,21]$. However, the optimal classifier trained with limited samples cannot meet the requirements of high precision, and so the whole SVM classifier can be constructed. By using ensemble learning theory, the generalization performance of the final classifier is improved by constructing multiple independent sub-classifiers.

The implementation of ensemble SVM depends on two factors: how to construct each member classifier and how to fuse the member classifier to form a strong classifier. In this study, we first selected 30 images as training data. For each image, we only extracted the features of the golden standard image region and its morphological extension region. Three-quarters of the data were used as training data; the rest were used as test data to evaluate the effect of classifiers. In this algorithm, the optimal number of members of the integrated classifier was not studied. There are four MRI data modes (Table 1), so we built the ensemble SVM classifier with eight members. For each classifier, we used a bagging-based random sampling method [22] to obtain random samples. To form an integrated SVM classifier, we employed the AdaBoost algorithm. The algorithm flow is as follows:

Step 1. Initial sample weight $w_{i}(t)=1 / N, \quad t=1,2,3 \cdots 8, N=8$

Step 2. For $\mathrm{t}=1-8$, if:

The classifier is defined as $f_{j}(j=1,2, \cdots N)$, then the classifier $f_{\mathrm{s}}$ that corresponds to the minimum weighted error, $\varepsilon_{s}$, must be found. The weighted error is defined as $\varepsilon_{j}=\sum_{i} w_{i}\left|f_{j}\left(x_{i}\right)-y_{i}\right|$, and $f_{j}\left(x_{i}\right)$ is the output of the subclass $f_{j}$ when the feature vector $x_{i}$ is the input, and $y_{i}$ is the standard value. After this loop is complete, $f_{t}=f_{s}$. Let $\alpha_{t}=(1 / 2) \ln \left[\left(1-\varepsilon_{t}\right) / \varepsilon_{t}\right]$, and then update the weight: $w_{t+1}(i)=w_{t}(i) \exp \left(-\alpha_{t} y_{i} f_{t}\left(x_{i}\right)\right)$. Normalize the value of $w_{t+1}$.

End.

Step 3. The final weight value is the output.

The details of constructing the ensemble SVM classifier are summarized in Fig. 3. After the ensemble classifier is obtained, it can be used for classification tasks, as illustrated on the right side of Fig. 3. 


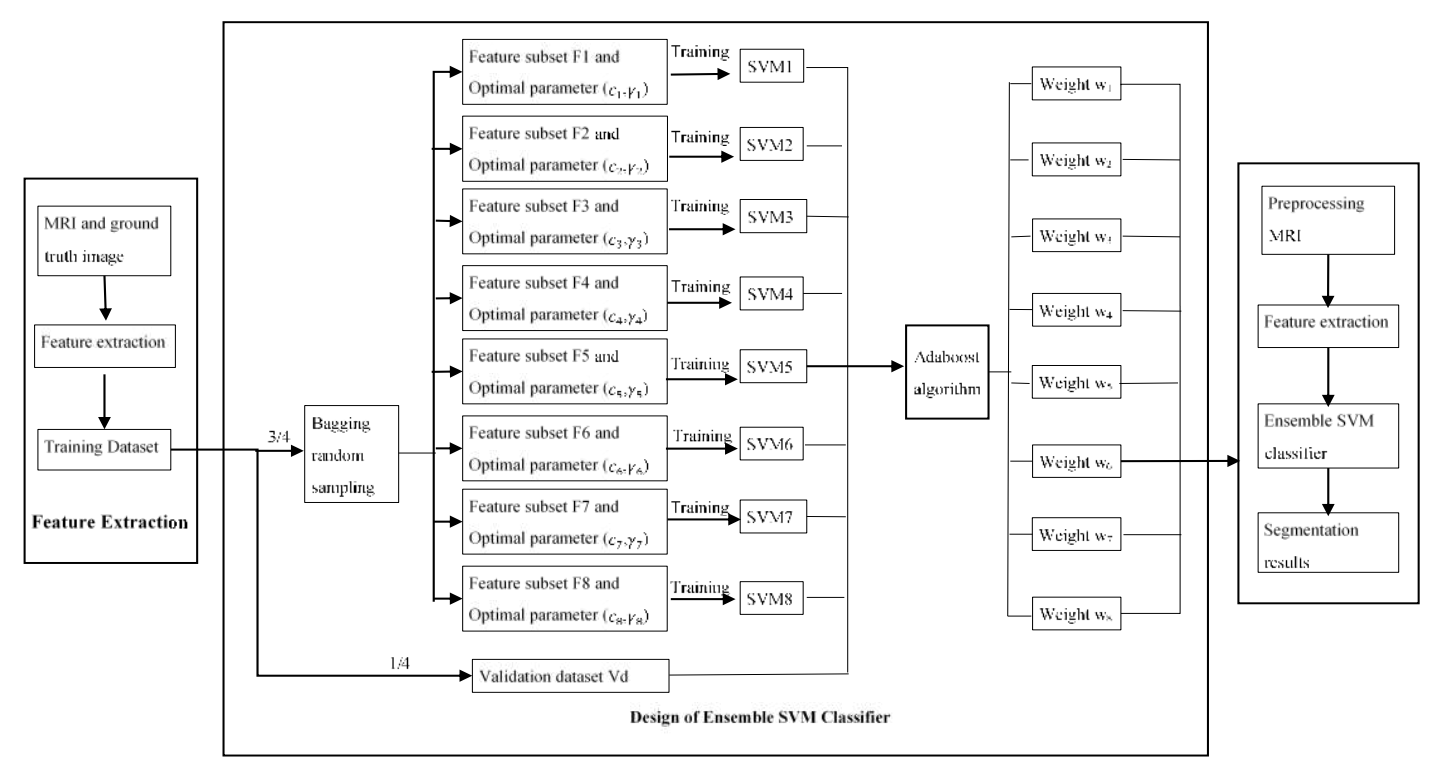

Fig 3. Diagram of brain tumor segmentation based on gray-level co-occurrence matrix (GLMC) texture features and a Support Vector Machine (SVM) model.

\subsection{Feature ranking and selection}

An important part of our study was to evaluate the influence of GLMC texture on brain tumor image segmentation. To this end, the effect of each GLMC texture component on image segmentation should be observed. Thus, we sorted the GLMC texture that participated in the construction of the classifier. Furthermore, the uncorrelated variables in the extracted features will slow down the calculation speed in the training and testing process. They may even cause some disturbing effects. We proposed an effective feature ranking and selection method to eliminate the irrelevant variables from the 112 extracted features presented in Table 1. Wang et al. [23] successfully applied SVM-RFE for screening medical image features. The main idea of the RFE method is to repeatedly establish an SVM model and then select the best features based on the coefficients. The specific process is as follows.

Step 1. Suppose there are two sets, one is $F S$, which contains all 112 feature sets, and the other is $R S$, which contains sorting features. At the beginning, $R S$ is an empty set.

Step 2. One feature in $R S$ is deleted, and the remaining 111 features are used to train the SVM classifier.

The classifier is initialized by empirical parameters to calculate the $D S$. If we repeat this procedure for all 112 features, we will get a set of $D S$ data. The feature corresponding to the maximum $D S$ value is the feature that contributes the least to the classifier. It will be moved from the $F S$ to the $R S$ set. After the first feature is selected, the second feature is chosen from the remaining 111 using the same method. The second feature is also placed in the $R S$ set after the first feature. Repeat the above process until $F S$ is empty.

The sorting index of the features selected by each member classifier is shown in Table 2. Notably, each member classifier contained different features due to the randomized training dataset. However, some features had strong discriminating power, namely 73, 101, 45, 17, 74, 102, 46, 18, 97, 13, 69, and 41. These features are top ranked in Table 2. The features 73, 101, 45, and 17 represent the range of the maximal correlation coefficient in the four modalities. The features $74,102,46$, and 18 represent the 
range of information measure of correlation in the four modalities. The features 97, 13, 69, and 41 are the mean of information measure of correlation in the four modalities. For the first 45 features, we found maximal correlation coefficient, information measure of correlation, Angular Second Moment, sum of squares, difference variance, contrast, and inverse difference moment (Table 2). This result is different from the application of the GLCM texture in other types of image processing. In most cases, Angular Second Moment, information measure of correlation, contrast, and entropy are used. However, in MRI tumor segmentation, maximal correlation coefficient, information measure of correlation (horizontal direction), Angular Second Moment, and sum of squares play an important role. Sum variance and entropy are sorted at the end. This result is beneficial to our subsequent feature selection.

Table 2. Optimal feature subsets and voting weights for each member classifier after training.

\begin{tabular}{|c|c|c|c|c|c|c|c|c|c|c|c|c|c|c|c|c|}
\hline \multirow{2}{*}{$\begin{array}{l}\text { Member } \\
\text { classifiers }\end{array}$} & \multicolumn{15}{|c|}{ Ranked feature indices (top 45 features) } & \multirow[t]{2}{*}{ Weight } \\
\hline & 73 & 101 & 45 & 17 & 74 & 102 & 46 & 18 & 13 & 97 & 69 & 41 & 81 & 109 & 25 & \\
\hline \multirow[t]{3}{*}{$\mathrm{SVM}_{1}$} & 14 & 98 & 53 & 70 & 42 & 89 & 5 & 33 & 61 & 110 & 82 & 26 & 54 & 11 & 12 & 0.1270 \\
\hline & 95 & 96 & 40 & 39 & 77 & 49 & 67 & 68 & 105 & 78 & 21 & 50 & 15 & 16 & 106 & \\
\hline & 73 & 101 & 45 & 17 & 74 & 102 & 46 & 18 & 13 & 97 & 69 & 41 & 109 & 81 & 25 & \\
\hline \multirow[t]{3}{*}{$\mathrm{SVM}_{2}$} & 14 & 98 & 53 & 70 & 42 & 89 & 5 & 33 & 61 & 110 & 82 & 26 & 54 & 40 & 39 & 0.1128 \\
\hline & 11 & 12 & 77 & 96 & 68 & 67 & 95 & 49 & 105 & 78 & 21 & 50 & 106 & 43 & 15 & \\
\hline & 73 & 101 & 17 & 45 & 74 & 102 & 46 & 18 & 97 & 13 & 69 & 41 & 109 & 81 & 25 & \\
\hline \multirow[t]{3}{*}{$\mathrm{SVM}_{3}$} & 14 & 98 & 53 & 70 & 42 & 89 & 5 & 33 & 61 & 110 & 82 & 26 & 54 & 11 & 40 & 0.1106 \\
\hline & 12 & 96 & 95 & 39 & 77 & 49 & 67 & 68 & 105 & 21 & 78 & 50 & 106 & 15 & 16 & \\
\hline & 73 & 101 & 45 & 17 & 74 & 102 & 46 & 18 & 13 & 97 & 69 & 41 & 109 & 81 & 25 & \\
\hline \multirow[t]{3}{*}{$\mathrm{SVM}_{4}$} & 14 & 98 & 53 & 70 & 42 & 5 & 89 & 33 & 61 & 110 & 82 & 26 & 54 & 40 & 39 & 0.1372 \\
\hline & 11 & 12 & 96 & 77 & 95 & 49 & 67 & 68 & 105 & 78 & 21 & 50 & 106 & 43 & 15 & \\
\hline & 73 & 101 & 45 & 17 & 74 & 102 & 46 & 18 & 13 & 97 & 69 & 41 & 81 & 109 & 25 & \\
\hline \multirow[t]{3}{*}{$\mathrm{SVM}_{5}$} & 14 & 98 & 53 & 70 & 42 & 89 & 5 & 33 & 61 & 110 & 82 & 26 & 54 & 40 & 39 & 0.1192 \\
\hline & 11 & 12 & 95 & 96 & 77 & 67 & 68 & 49 & 105 & 78 & 21 & 50 & 15 & 106 & 16 & \\
\hline & 73 & 101 & 45 & 17 & 74 & 102 & 46 & 18 & 13 & 97 & 69 & 41 & 109 & 81 & 25 & \\
\hline \multirow[t]{3}{*}{$\mathrm{SVM}_{6}$} & 14 & 53 & 98 & 70 & 42 & 89 & 5 & 33 & 61 & 110 & 82 & 26 & 54 & 11 & 12 & 0.1288 \\
\hline & 39 & 40 & 96 & 95 & 77 & 49 & 67 & 68 & 105 & 78 & 21 & 50 & 106 & 15 & 16 & \\
\hline & 73 & 101 & 17 & 45 & 74 & 102 & 46 & 18 & 13 & 97 & 69 & 41 & 81 & 109 & 25 & \\
\hline \multirow[t]{3}{*}{$\mathrm{SVM}_{7}$} & 14 & 98 & 53 & 70 & 42 & 89 & 5 & 33 & 61 & 110 & 82 & 26 & 54 & 40 & 39 & 0.1343 \\
\hline & 11 & 12 & 96 & 77 & 67 & 68 & 95 & 49 & 105 & 78 & 21 & 50 & 106 & 43 & 44 & \\
\hline & 73 & 101 & 45 & 17 & 74 & 102 & 46 & 18 & 13 & 97 & 69 & 41 & 109 & 81 & 25 & \\
\hline \multirow[t]{2}{*}{$\mathrm{SVM}_{8}$} & 14 & 98 & 53 & 70 & 42 & 5 & 89 & 33 & 61 & 82 & 110 & 26 & 54 & 11 & 12 & 0.1301 \\
\hline & 40 & 39 & 96 & 95 & 77 & 49 & 67 & 68 & 105 & 78 & 21 & 50 & 106 & 15 & 16 & \\
\hline
\end{tabular}




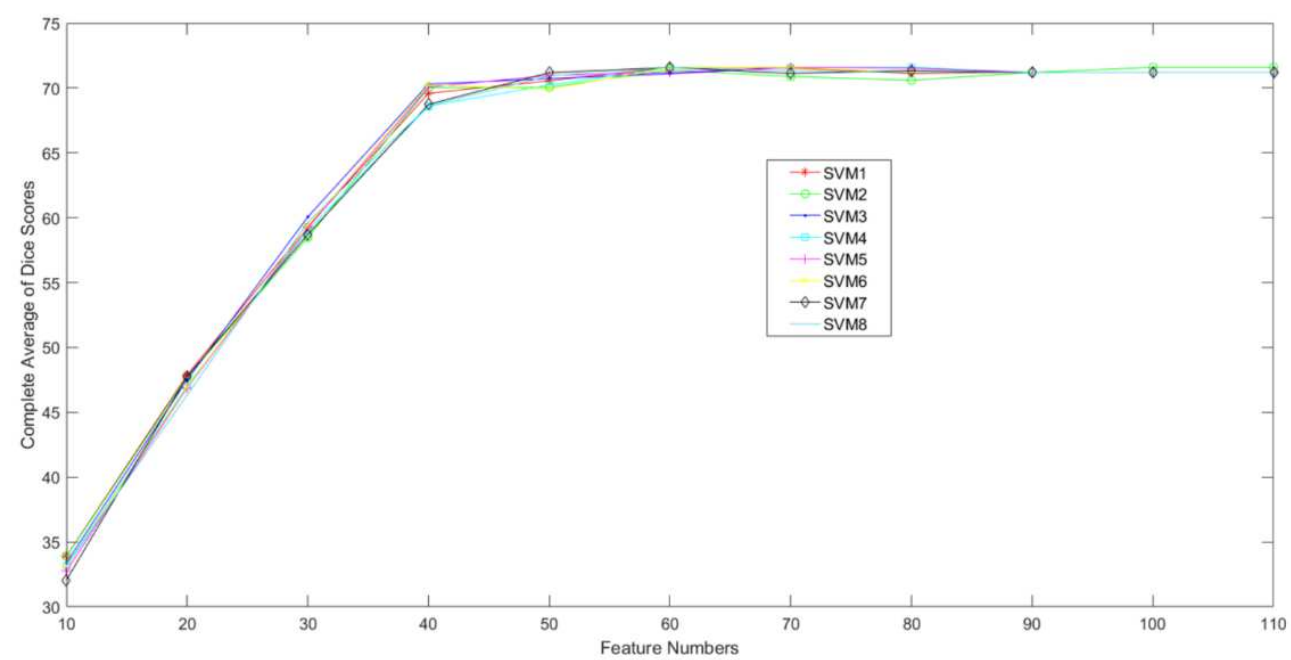

Fig. 4. Dice similarity coefficient (DSC) of member classifiers as functions of the number of features included in each classifier.

Fig. 4 presents the relationship between the number of features and the DSC of the complete segmentation. Here, the DSC was calculated based on the validation data set. DSC was significantly increased when the number of features was less than 60 . When the number of features was approximately 60 , the DSC of each classifier reached a peak of $71.6 \%$. When the number of feature selections exceeded 60 , the DSC curve tended to be flat and even decreased slightly. When all 112 features were selected, the DSC was $71.2 \%$. These data demonstrated that when designing the classifier more features are not necessarily better. The selection of certain features decreases the accuracy of segmentation. In the following experiments, $60 \mathrm{GLCM}$ features were selected to design a classifier. From the experimental results, when GLCM features were used alone, the segmentation accuracy was not high. In the final algorithm, GLCM features and some grayscale features were selected. These grayscale features included voxel grayscale values, grayscale mean values and grayscale variances on three different spatial scales. There are seven grayscale features. For MRI data, there are four modalities, so there are a total of 88 features here.

\section{Results}

3.1 Training and validation data

The online MR brain tumor data library Brain Tumor Image Segmentation Benchmark 2015 (BraTs2015) was used in experiments. In the database, T1, T2, T1ce, and FLAIR images for each patient are available. All images have been registered. Each modal image was linearly aligned according to the human body standard brain, and the pixel points correspond to each other. The three-dimensional size of each modal MRI image was $240 \times 240 \times 155$, and the true value label is the result of manual calibration by multiple experts. In this paper, DSC scores were used to evaluate the segmentation results of brain tumors. The similarity coefficient indicates the degree of similarity between the experimental segmentation result and the label.

\subsection{Segmentation result}

MR images from 100 patients were randomly selected as training sets. We evaluated the final model on 30 patients. In the training phase, the image area around the gold standard was selected. In the test phase, we performed pre-segmentation. In image segmentation, we segmented five different labels: one 
normal and four tumor types, including normal brain, necrosis, edema, non-enhancing tumor, and enhancing tumor (Table 3). Overall, the results of the training data were better than the test data.

Table 3. Dice similarity complex (DSC) scores obtained from the BraTS2015 training set and the testing set for different tumor tissues.

\begin{tabular}{|c|c|c|c|c|c|c|c|c|}
\hline Schedule & Capacity & & Necrosis & Edema & $\begin{array}{c}\text { Non- } \\
\text { enhancing }\end{array}$ & Enhancing & Core & Complete \\
\hline \multirow[t]{4}{*}{ Training set } & Low-grade & median & 46.3 & 88.6 & 65.7 & 47.6 & 93.5 & 93.2 \\
\hline & & average & 48.4 & 77.3 & 68.5 & 45.4 & 92.7 & 92.1 \\
\hline & High-grade & median & 75.2 & 85.2 & 46.2 & 82.8 & 87.3 & 96.9 \\
\hline & & average & 76.5 & 86.5 & 47.7 & 84.1 & 86.8 & 95.3 \\
\hline \multirow[t]{4}{*}{ Testing set } & Low-grade & median & 18.2 & 65.4 & 62.7 & 44.5 & 88.2 & 90.8 \\
\hline & & average & 22.8 & 67.2 & 63.2 & 38.7 & 87.8 & 91.2 \\
\hline & High-grade & median & 69.7 & 78.2 & 44.1 & 74.8 & 89.0 & 92.1 \\
\hline & & average & 68.2 & 80.1 & 46.7 & 76.4 & 88.6 & 92.4 \\
\hline
\end{tabular}

With the training data, for low-grade tumors, we obtained a $92.1 \%$ average DSC for segmenting the complete tumor region. For high-grade tumors, the average was slightly higher at $95.3 \%$. For low-grade tumors, the DSC for segmentation of edema and non-enhanced tumor tissue was good: $77.3 \%$ and $68.5 \%$, respectively. Segmentation results for high-grade tumors were better compared to low-grade tumors. For necrotic and enhanced tissues, the segmentation accuracy of this method was not very good; the results of high-grade tumors were better. This difference is because necrosis or enhancement usually does not show in low-grade tumors. The proportion of these areas in the image is small, and these areas are easily mistaken for other tumor tissues. For high-grade tumors, we obtained an average DSC of $86.5 \%, 84.1 \%$, and $86.8 \%$ for edema, enhancing tissue, and core tissue, respectively. The worst results were from segmentation of the non-enhancing tissue, with an average DSC of $46.2 \%$.

The method did not perform as well for the testing data compared to the training set. For low-grade tumors, the average DSC for the core tumor was $87.8 \%$. The average DSC of the complete tumor was $91.2 \%$. These values increased to $88.6 \%$ and $92.4 \%$ in high-grade tumors. The segmentation accuracy was the worst for necrotic and enhanced tissues; the average DSCs were $22.8 \%$ and $38.7 \%$, respectively. Necrosis or enhancement tissues usually do not show in low-grade tumors and there are not many samples to train SVM model. For high-grade tumors, the average DSC of edema, enhanced tissue, and non-enhanced tissue were $80.1 \%, 76.4 \%$ and $46.1 \%$, respectively. Overall, our data demonstrated the method is better for high-grade tumor segmentation. An example of low-grade tumor segmentation is shown in Fig. 5, whereas Fig. 6 presents an example of high-grade tumor segmentation.

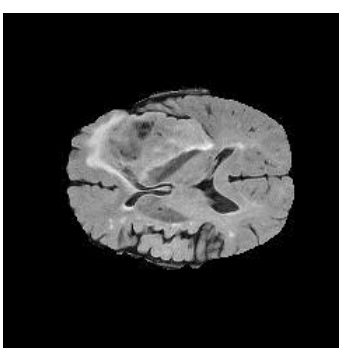

(a)

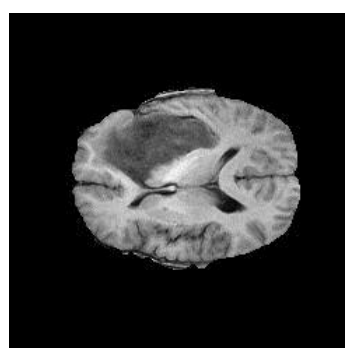

(b)

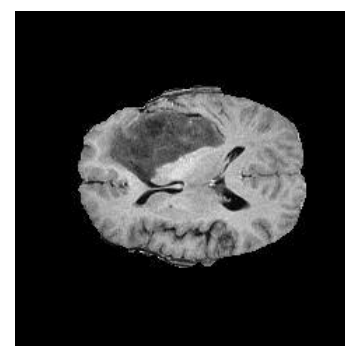

(c)

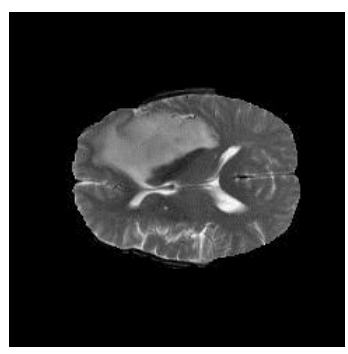

(d) 


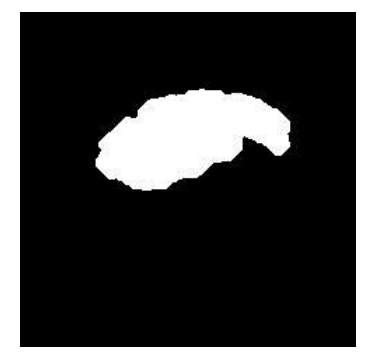

(e)

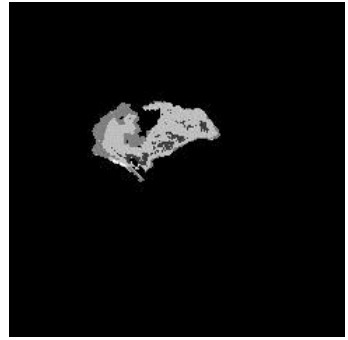

(f)

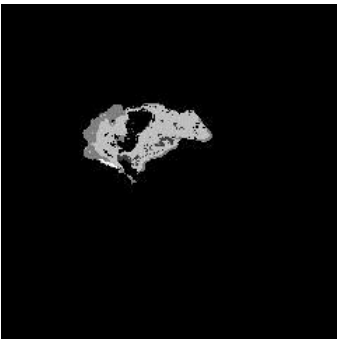

(g)

Fig5. Low-grade image segmentation

(a) FLAIR image (b) T1 image (c) T1C image (d) T2 image(e) Pre-segmented image (f) Final segmentation image (g) Ground-truth image

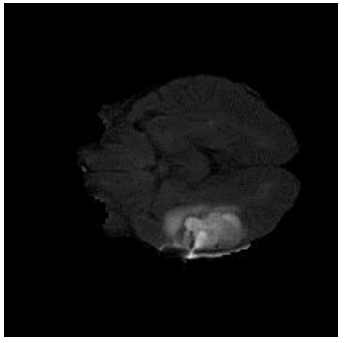

(a)

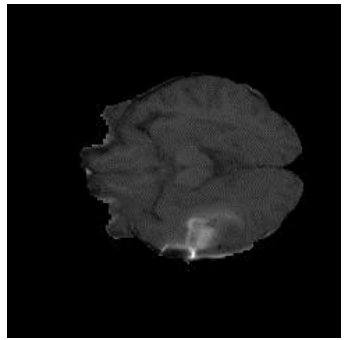

(b)

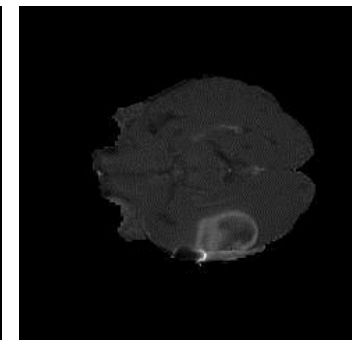

(c)

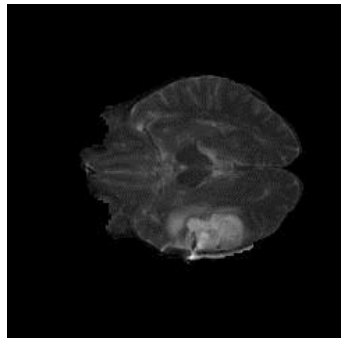

(d)

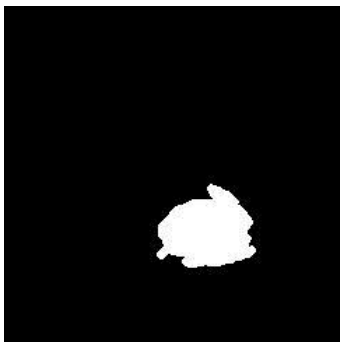

(e)

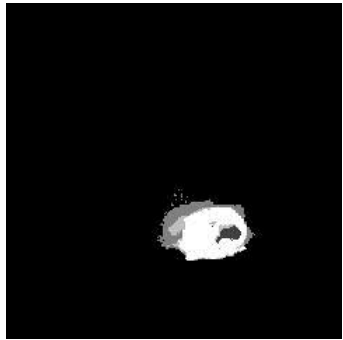

(f)

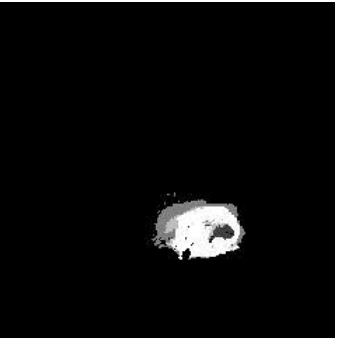

(g)

Fig6. Low-grade image segmentation

(b) FLAIR image (b) T1 image (c) T1C image (d) T2 image(e) Pre-segmented image (f) Final segmentation image (g) Ground-truth image

\section{Discussion}

In this study, a GLCM texture-based brain tumor segmentation method was evaluated. The SVM-RFE was used to determine which components of GLCM texture were most useful for segmentation. One-hundred-twelve GLMC texture features were sorted using SVM-RFE. According to the sorting result, 60 important features were selected. Among these 60 features are maximal correlation coefficient, information measure of correlation, Angular Second Moment, sum of squares, difference variance, and inverse difference moment. In many applications of GLMC texture, entropy is often used, but it was not important in brain tumor segmentation. The same is true for contrast. In feature sorting, they seldom appear in the front position. In future research, we will focus on the above six components of GLCM texture. These components can be combined with other texture expressions (such as Tamura texture) to represent brain tumor image information. The method of fusing multiple textures will be studied in the future. 
In this paper, we built an ensemble SVM classifier that comprised eight trained single classifiers. Based on the DSC value of complete tumor segmentation, we set a weight value for each classifier. Our segmentation results were better than previous studies [23,24], which used a single classifier. This improvement was due to a pre-segmentation process and ensemble SVM classifier. However, there was still a gap between our method and the algorithm based on convolutional neural network (CNNs)[25,26]. When these methods are trained, many samples and extensive expertise are required to ensure proper convergence. A previously proposed method only used T1 MR images [27]. The average DSC value of complete segmentation was only $85.7 \%$ for gliomas. In the case of a small amount of data, the performance of the CNN-based method is also general. In the process of clinical diagnosis, we cannot get sufficient MR data. In our method, GLMC texture and gray features are extracted as classifier features, and the amount of data required is not particularly large. The whole training process is not very complicated. If more features are extracted, the segmentation accuracy will be improved. In our method, we set the value of $d$ as 5 . The spatial context of a voxel is $5 \times 5$. A larger $d$ might generate better results. However, this factor was not considered due to the increased computational complexity. Additionally, we only segmented one model, namely low-grade and high-grade tumors, whereas previous studies usually design two models. In clinical practice, it is not always known a priori which tumor type to analyze.

\section{Conclusion}

The precise segmentation of brain tumors is the most important and crucial step in their diagnosis and treatment. In future research, we will focus on the six components of GLCM texture, maximal correlation coefficient, information measure of correlation, Angular Second Moment, sum of squares, difference variance, and inverse difference moment. These components can be combined with other texture expressions (such as Tamura texture) to represent brain tumor image information. The method of fusing multiple textures will be studied in the future.

\section{Abbreviations}

MRI: Magnetic resonance imaging.

GLCM: Gray-level co-occurrence matrix.

SVM: Support vector machine.

CNN: Convolutional neural network.

DSC: Dice similarity coefficient.

\section{Declarations}

\subsection{Ethics approval and consent to participate}

This article does not contain any studies with human participants or animals performed by any of the authors.

\subsection{Consent for publication}

I would like to declare on behalf of my co-authors that the work described was original research that has not been published previously, and not under consideration for publication elsewhere, in whole or in part.

7.3 Competing interests

The authors declare that they have no known competing financial interests or personal relationships that could have appeared to influence the work reported in this paper. 


\subsection{Funding}

This work was supported by the Fundamental Research Funds for the Central Universities (CZQ19005).

\subsection{Authors' contributions}

ZY contributed to the conception of the study.NL performed the experiment.NL, ZY performed the data analyses and wrote the manuscript.

\subsection{Acknowledgements}

This thesis would not have been possible without the consistent and valuable reference materials that I received from Professor Zhiyong Xiong, whose insightful guidance and enthusiastic encouragement in the course of my shaping this thesis definitely gamy deepest gratitude.

\section{Availability of data and materials}

The data that support the findings of this study are available from the website. https://www.smir.ch/BRATS/Start2015.

\section{References}

[1] Mohan G, Subashini M, MRI based medical image analysis: survey on brain tumor grade classification, Biomedical Signal Processing and Control, 2018; 39(1): 139-161.

[2] Torheim T, et al. Classification of dynamic contrast enhanced MR images of cervical cancers using texture analysis and support vector machine. IEEE Transactions on Medical Imaging, 2014; 33(8): 1648-1656.

[3] Mougiakakou SG, et al. Differential diagnosis of CT focal liver lesions using texture features, feature selection and ensemble driven classifiers. Artificial Intelligence in Medicine, 2007; 41(1): 25-37.

[4] Chen X, et al. Differentiation of true-progression from pseudo progression in glioblastoma treated with radiation therapy and concomitant temozolomide by GLCM texture analysis of conventional MRI. Clinical Imaging, 2015; 39(5): 775-780.

[5] Vamvakas A, et al. Imaging biomarker analysis of advanced multiparametric MRI for glioma grading. Physica Medica, 2019; 60: 188-198.

[6] Vallabhaneni RB, Rajesh V. Brain tumor detection using mean shift clustering and GLCM features with edge adaptive total variation denoising technique. Alexandria Engineering Journal, 2018; 57(4): 2387-2392.

[7] Abraham B, Nair MS. Computer-aided classification of prostate cancer grade groups from MRI images using texture features and stacked sparse auto encoder. Computerized Medical Imaging and Graphics, 2018; 69: 60-68.

[8] Chakraborty J, Midya A, Mukhopadhyay S. Detection of the nipple in mammograms with Gabor filters and the Radon transform. Biomedical Signal Processing and Control, 2015; 15: 80-89

[9] Hackmack K, Friedemann P, Weygandt M. Multi-scale classification of disease using structural MRI and wavelet transform. NeuroImage, 2012; 62(1): 48-58.

[10] Haralick RM, Shanmuga K, Dinstein I. Texture features for image classification. IEEE Transactions on Systems, Man, and Cybernetics, 1973; 23: 610-621.

[11] Gonzalez R, Woods R. Digital image processing. London: Pearson Education; 2011.

[12] Mayerhoefer ME, et al. Effects of MRI acquisition parameter variations and protocol heterogeneity on the results of texture analysis and pattern discrimination: an application-oriented study. Medical Physics, 2009; 36(4): $1236-1243$.

[13] Alic L, et al. Heterogeneity in DCE-MRI parametric maps: a biomarker for treatment response? Physics in Medicine and Biology, 2011; 56(6): 1601-1616. 
[14] Agner, SC, et al. Textural kinetics: a novel dynamic contrast-enhanced (DCE)-MRI feature for breast lesion classification. Journal of Digital Imaging, 2011; 24(3): 446-463.

[15] Torheim T, et al. Classification of dynamic contrast enhanced MR images of cervical cancers using texture analysis and Support Vector Machines. IEEE Transactions on Medical Imaging, 2014; 33(8): 1648-1656.

[16] Bonte S, Goethals I, Van Holen R. Machine learning based brain tumour segmentation on limited data using local texture and abnormality. Computers in Biology and Medicine, 2018; 98: 39-47.

[17] Wang R, et al. Automatic blur type classification via ensemble SVM. Signal Processing: Image Communication, 2019, 71: 24-35

[18] Li DY, Li WF, Liao QM. Based on the asymmetric information and active contour model of brain tumor segmentation system. Journal of Tsinghua University (Science and Technology), 2013; 53(7): 995-1000.

[19] Vapnik V. The nature of statistics learning theory. New York: Springer Verlag; 1995.

[20] Song B, Wang H, Wei R. Brain tumor segmentation of magnetic resonance imaging based on improved Support Vector Machines. Journal Of Medical Imaging And Health Informatics, 2019; 9(5): 1011-1016.

[21] Sengupta AU, et al. On differentiation between vasogenic edema and non-enhancing tumor in high-grade glioma patients using a support vector machine classifier based upon pre and post-surgery MRI images. European Journal of Radiology, 2018; 106: 199-208.

[22] Guyon I, et al. Gene selection for cancer classification using support vector machines, Machine Learning, 2002; 46(1-3): 389-422.

[23] Wang R , Li R , Lei Y , et al. Tuning to optimize SVM approach for assisting ovarian cancer diagnosis with photoacoustic imaging[J]. Bio-Medical Materials and Engineering, 2015; 26(s1):S975-S981

[24] Zhang L, et al., Fast multi-view, segment graph kernel for object classification. Signal Processing, 2013; 93(6): 1597-1607.

[25] Kamnitsas K, et al. Efficient multi-scale 3D CNN with fully connected CRF for accurate brain lesion segmentation, Medical Image Analysis, 2017; 36: 61-78.

[26] Havaei M, et al. Brain tumor segmentation with deep neural networks, Medical Image Analysis, 2017; 35: 1831 .

[27] Kaldera HNTK, Gunasekara SR, Dissanayake MB. Brain tumor classification and segmentation using Faster R-CNN. 2019 Advances in Science and Engineering Technology International Conferences (ASET), Dubai, United Arab Emirates; 2019, 1-6. 


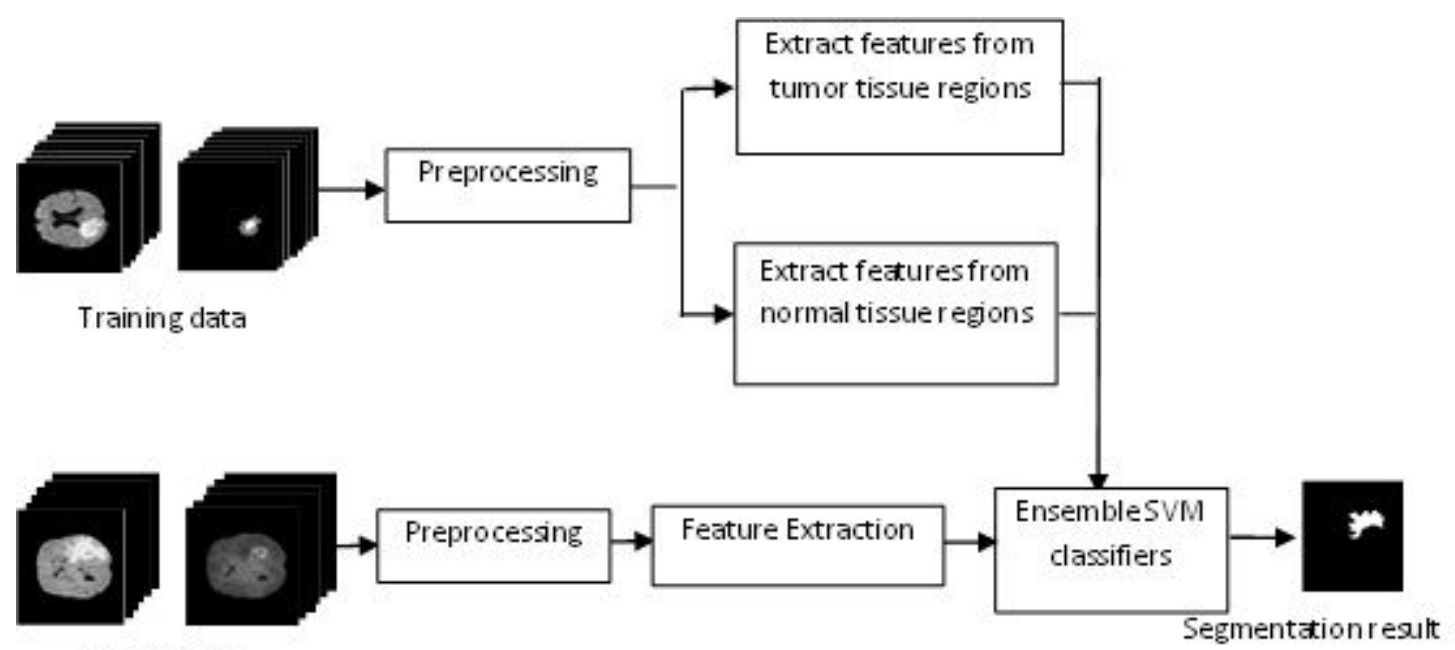

Testing data

Figure 1

The workflow of image segmentation.

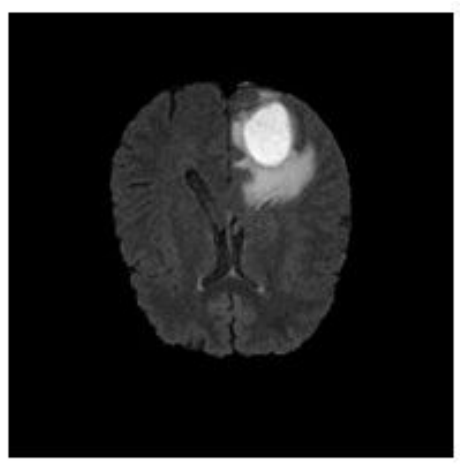

(a)

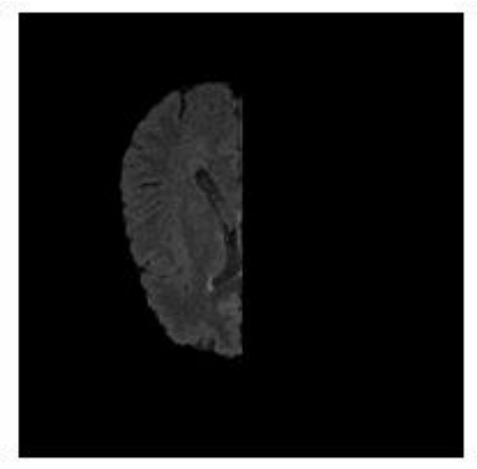

(b)

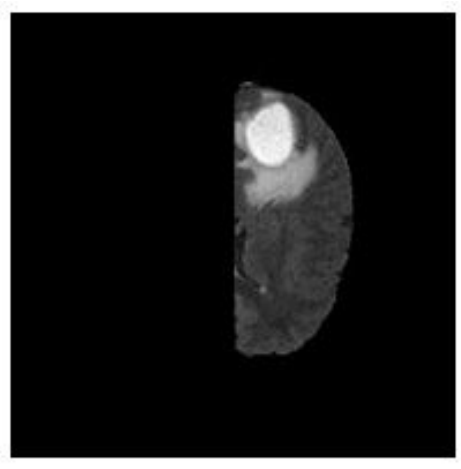

(c)

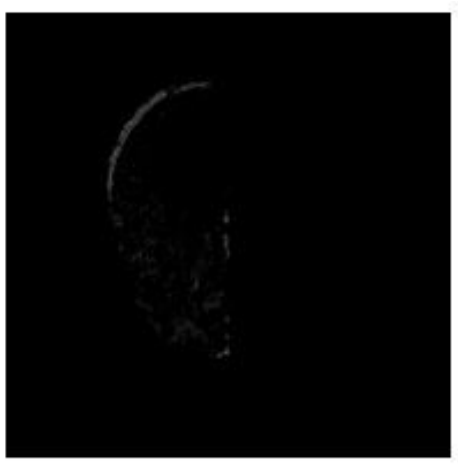

(d)

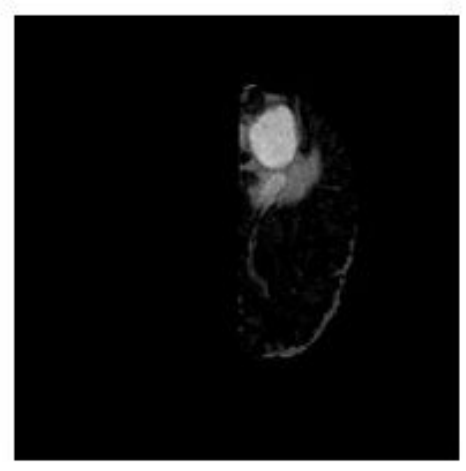

(e)

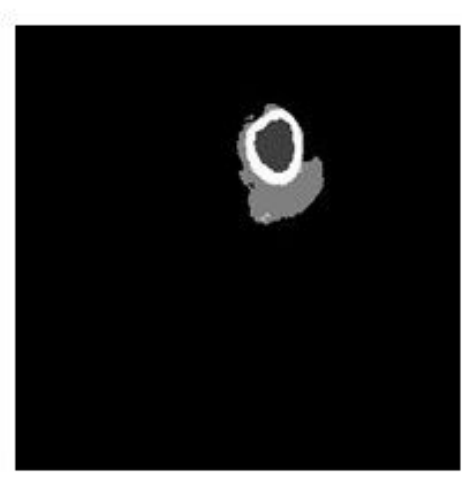

(f)

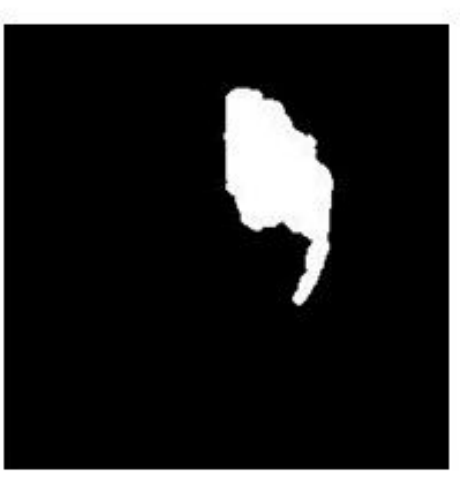

(g)

\section{Figure 2}

Symmetric pre-segmentation of brain tumor images: (a) original image, (b) left brain image, (c) right brain image, (d) image, (e) image, (f) ground-truth image, and (g) pre-segmented image of all sequences in MRI. 


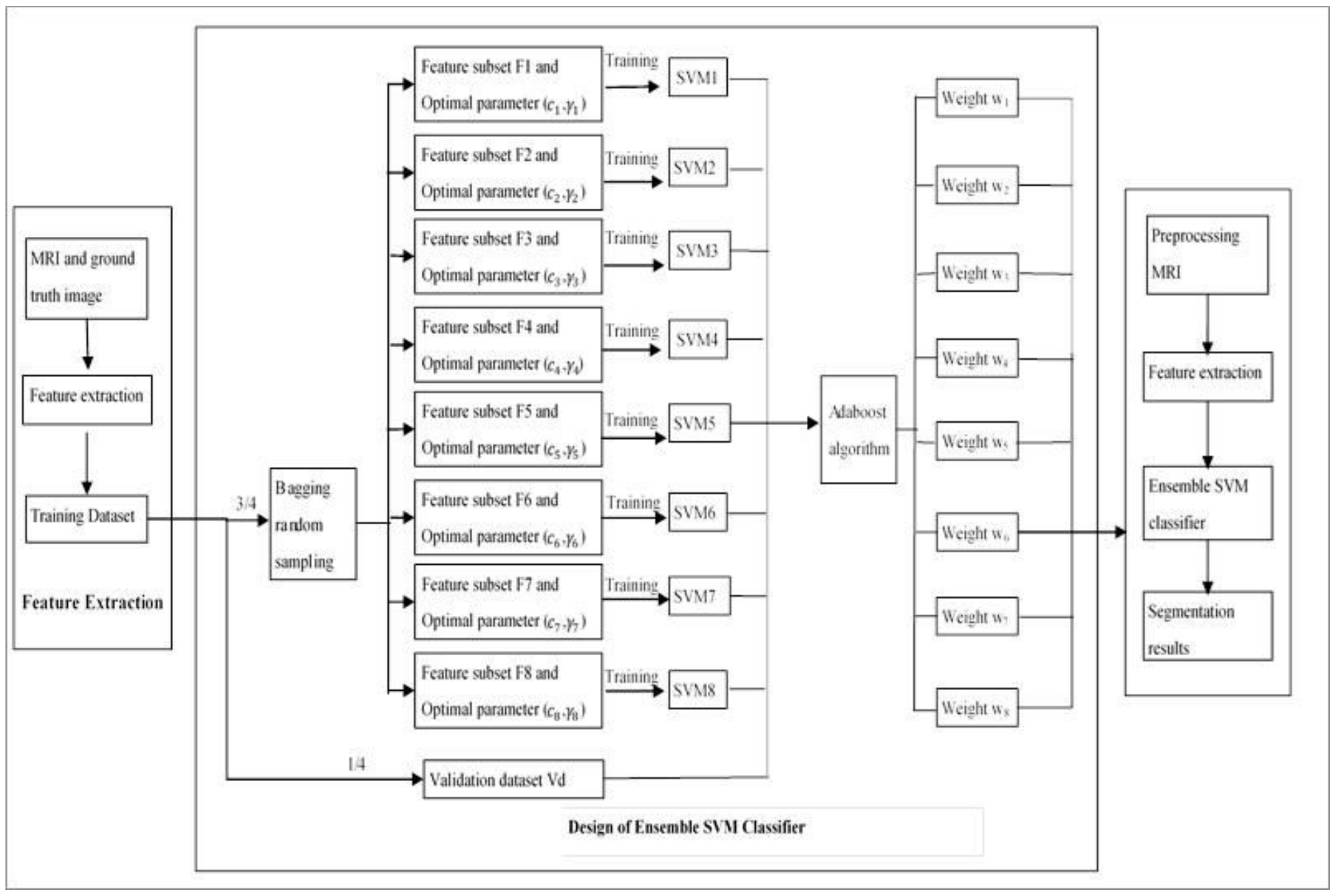

Figure 3

Diagram of brain tumor segmentation based on gray-level co-occurrence matrix (GLMC) texture features and a Support Vector Machine (SVM) model. 


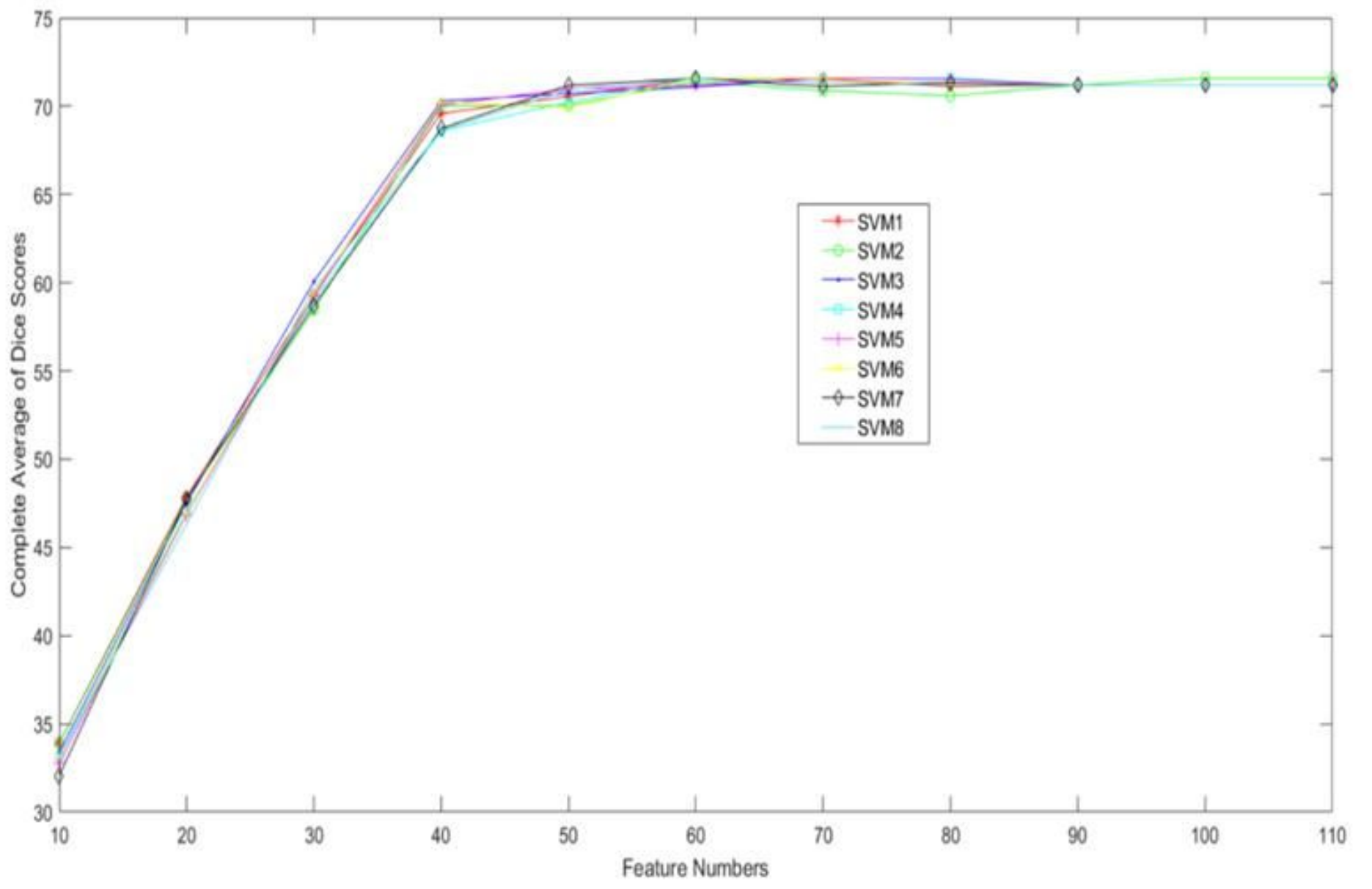

Figure 4

Dice similarity coefficient (DSC) of member classifiers as functions of the number of features included in each classifier. 


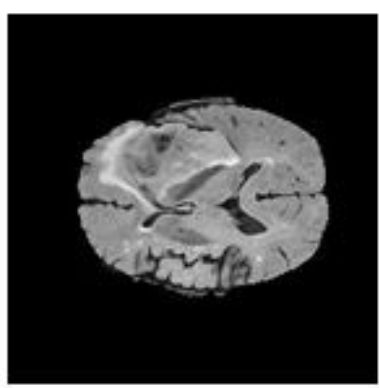

(a)

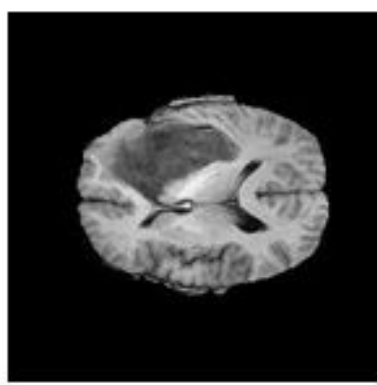

(b)

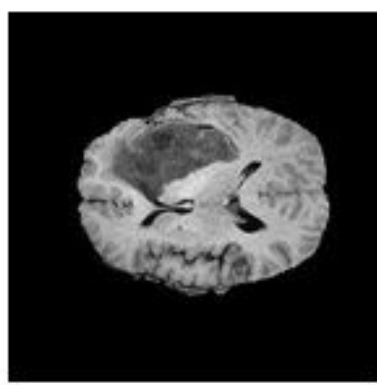

(c)

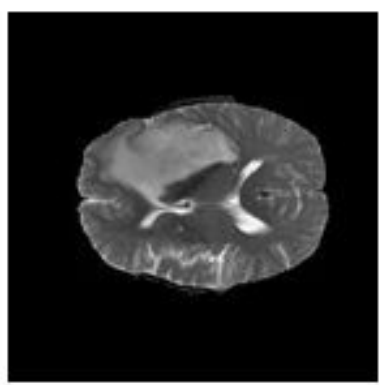

(d)

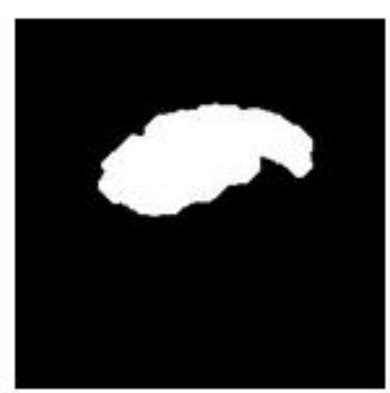

(e)

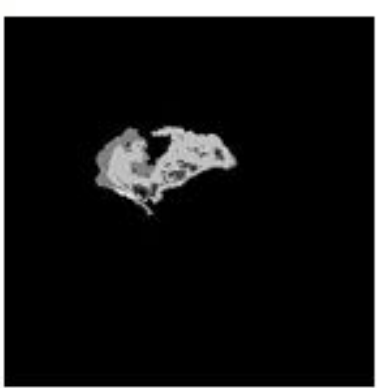

(f)

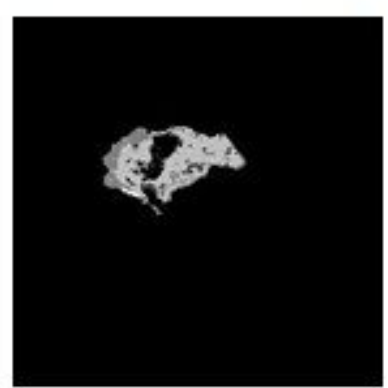

(g)

\section{Figure 5}

Low-grade image segmentation (a) FLAIR image (b) T1 image (c) T1C image (d) T2 image(e) Presegmented image (f) Final segmentation image (g) Ground-truth image

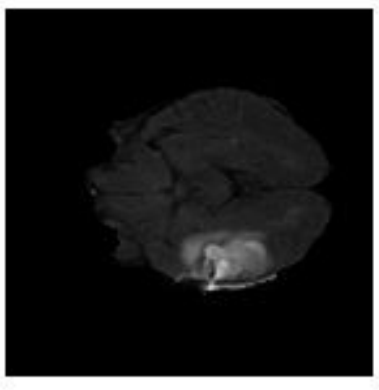

(a)

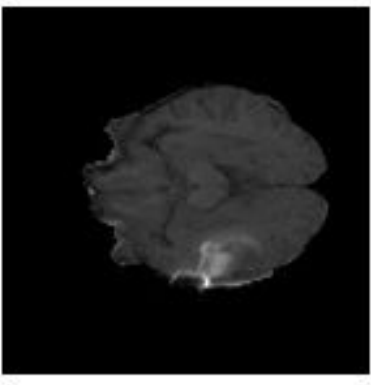

(b)

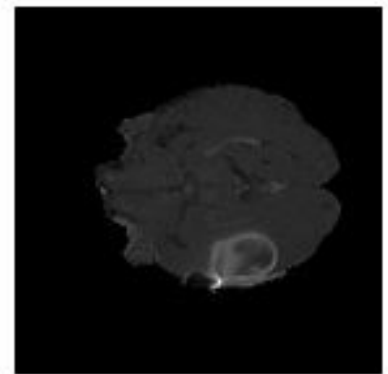

(c)

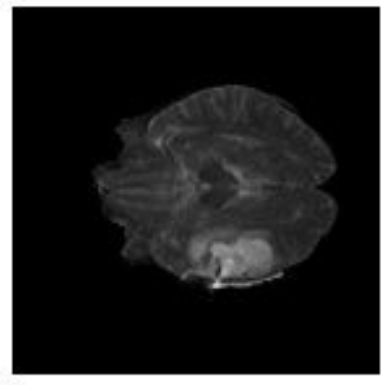

(d)

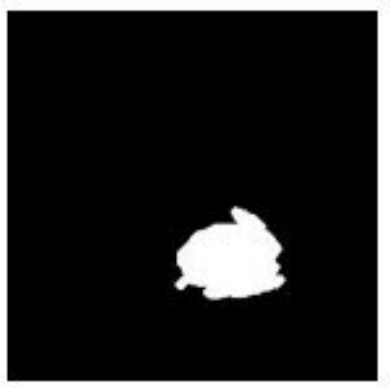

(e)

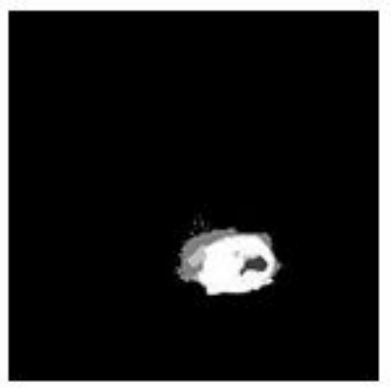

(f)

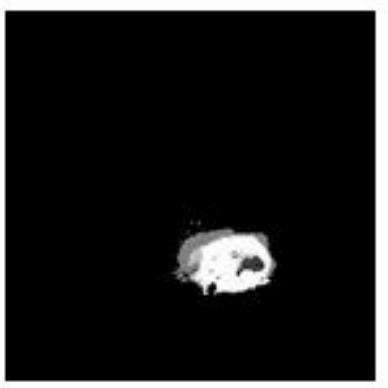

(g)

Figure 6 
Low-grade image segmentation (b) FLAIR image (b) T1 image (c) T1C image (d) T2 image(e) Presegmented image (f) Final segmentation image (g) Ground-truth image 